Lithuanian Mathematical Journal, Vol.X, No. x, 20xx, Author's Version May 27, 2013

\title{
EXACT TAIL ASYMPTOTICS OF THE SUPREMUM OF STRONGLY DEPENDENT GAUSSIAN PROCESSES OVER A RANDOM INTERVAL
}

\author{
Zhongquan Tan \\ College of Mathematics, Physics and Information Engineering, Jiaxing University, Jiaxing 314001, PR China \\ (e-mail: tzq728@163.com)

\section{Enkelejd Hashorva ${ }^{1}$} \\ Department of Actuarial Science, Faculty of Business and Economics,University of Lausanne, UNIL-Dorigny 1015 \\ Lausanne, Switzerland \\ (e-mail: enkelejd.hashorva@unil.ch)
}

Received

\begin{abstract}
Let $\mathcal{T}$ be a positive random variable independent of the real-valued stochastic process $\{X(t), t \geq 0\}$. In this paper we investigate the asymptotic behaviour of $P\left(\sup _{t \in[0, \mathcal{T}]} X(t)>u\right)$ as $u \rightarrow \infty$ assuming that $X$ is a strongly dependent stationary Gaussian process and $\mathcal{T}$ has a regularly varying survival function at infinity with index $\lambda \in[0,1)$. Under asymptotic restrictions on the correlation function $r(t)$ of the process, we show that $P\left(\sup _{t \in[0, \mathcal{T}]} X(t)>u\right)=$ $c^{\lambda} P(\mathcal{T}>m(u))(1+o(1))$ with $c$ some positive finite constant and $m(\cdot)$ defined in terms of the local behaviour of the correlation function and the standard Gaussian distribution.
\end{abstract}

Keywords: ...Gaussian processes; strong dependence; supremum over a random interval; exact tail asymptotics.

AMS Classification: Primary 60G15; secondary 60G70

\section{INTRODUCTION}

Consider $\{X(t), t \geq 0\}$ a standard (zero-mean and unit-variance) stationary Gaussian process with correlation function $r(t)$ and a.s. continuous sample paths. The investigation of the tail asymptotics of the supremum of Gaussian processes is a hard problem. Typically the study of the asymptotic tail behaviour of the supremum of the process on some finite interval $[0, T], T>0$, i.e., the asymptotics

$$
P\left(\sup _{t \in[0, T]} X(t)>u\right), \text { as } u \rightarrow \infty
$$

is easier to deal with compared to the case that $T=\infty$. The excellent monograph [28] provides the fundamental theoretical results for dealing with both cases under various assumptions on the correlation function and the variance function of the process. There are numerous research fields and many applications where the asymptotic expansion of the probability in (1.1) is crucial, see e.g., [1], [22], [28], [21], and [17]. With motivations from applications in queuing theory, insurance, and hydrodynamics [12], [35], [11], [25],

\footnotetext{
${ }^{1}$ Corresponding author
} 
[23], and recently [3, 4] consider instead of $T$ deterministic a positive random variable $\mathcal{T}$ being independent of the Gaussian process $X$.

In both [12] and [3] some general Gaussian processes $\{X(t), t \in[0, \infty)\}$ with stationary increments are considered. This paper is motivated by [4] where the Gaussian process is assumed to be stationary. We formulate next the assumptions and the main findings of the aforementioned article:

Suppose that the correlation function $r(t):=\operatorname{Cov}(X(s), X(s+t))$ satisfies

(A1). $r(t)=1-C_{*}|t|^{\alpha}+o\left(|t|^{\alpha}\right)$ as $t \rightarrow 0$, with $\alpha \in(0,2]$ and $C_{*}>0$;

(A2). $r(t) \log (t) \rightarrow 0$ as $t \rightarrow \infty$.

The positive random variable $\mathcal{T}$ which throughout this paper is supposed to be independent of the Gaussian process $\{X(t), t \in[0, \infty)\}$ has survival function $\bar{H}$ satisfying the following assumption:

(B). $\bar{H}$ is regularly varying at infinity with parameter $\lambda \in[0,1)$, i.e., $P(\mathcal{T}>t)=\bar{H}(t)=L(t) t^{-\lambda}$, where $L(\cdot)$ is slowly varying function at $\infty$.

For details on regularly varying and slowly varying functions see e.g., [6].

The key findings of [4] are collected in the following theorem:

Theorem 1. Let $\{X(t), t \geq 0\}$ be a standard stationary Gaussian process with correlation function satisfying (A1) and (A2) being further independent of $\mathcal{T}$. If $\bar{H}$ satisfies Assumption (B), then as $u \rightarrow \infty$

$$
P\left(\sup _{t \in[0, \mathcal{T}]} X(t)>u\right)=\Gamma(1-\lambda)\left(\frac{H_{\alpha} C_{*}^{1 / \alpha}}{\sqrt{2 \pi}}\right)^{\lambda} L\left(u^{\frac{\alpha-2}{\alpha}} \exp \left(u^{2} / 2\right)\right) u^{\frac{\lambda(2-\alpha)}{\alpha}} \exp \left(-\frac{\lambda u^{2}}{2}\right)(1+o(1 .)) \text { ) }
$$

where $H_{\alpha}$ is the Pickands constant and $\Gamma(\cdot)$ stands for the Euler gamma function.

In the literature Assumption (A2) is referred to as the weak dependence or the Berman's condition (see [5]) and consequently the stationary Gaussian process $\{X(t), t \in[0, \infty)\}$ is called a weakly dependent stationary Gaussian process. A natural generalisation of $(A 2)$ is the following assumption

(A3). $r(t) \log t \rightarrow r \in(0, \infty)$, as $t \rightarrow \infty$.

In analogy, the stationary Gaussian process $\{X(t), t \geq 0\}$ with correlation function satisfying Assumption (A3) is called strongly dependent. Indeed, condition (A3) is a natural extension of (A2). For related studies on extremes of strongly dependent Gaussian processes, we refer to [24], [28], [16], [18], [19], [30], [15], [31], [32],[33], [34].

The aim of this paper is to study the asymptotic behaviour of the supremum of strongly dependent stationary Gaussian processes over some random interval $[0, \mathcal{T}]$. Our results show that a similar asymptotic expansion as that obtained in Theorem 1 still holds for strongly dependent stationary Gaussian processes.

Brief organisation of the paper: We present the main result in Section 2 followed then by several auxiliary results and proofs displayed in Section 3.

\section{MAIN RESULT}

As mentioned in the Introduction [4] derived the exact asymptotics of the weakly dependent stationary Gaussian processes assuming the regular variation of the survival function $\bar{H}$ of $\mathcal{T}$, where in the asymptotics the prominent Pickands constant appears. The reason for that is the Pickands exact asymptotics (see [26]) of the supremum of the stationary Gaussian process $\{X(t), t \in[0, \infty)\}$ over the finite interval $[0, T], T>0$ given by

$$
P\left(\sup _{t \in[0, T]} X(t)>u\right)=T C_{*}^{1 / \alpha} H_{\alpha} u^{2 / \alpha} \Psi(u)(1+o(1)), \text { as } u \rightarrow \infty,
$$

provided that the correlation function of the Gaussian process satisfies Assumption (A1) and further $r(t)<$ 1 for all $t>0$. Here $\Psi(\cdot)=1-\Phi(\cdot)$ is the survival function of a $N(0,1)$ random variable. Pickands 
constant $H_{\alpha}$ is defined by

$$
H_{\alpha}=\lim _{x \rightarrow \infty} x^{-1} \mathbb{E}\left(\exp \left(\sup _{t \in[0, x]} Z(t)\right)\right),
$$

where $\{Z(t), t \geq 0\}$ is a fractional Brownian motion with continuous sample paths, mean function $\mathrm{e} Z(t)=$ $-t^{\alpha}$ and covariance function

$$
\operatorname{cov}(Z(s), Z(t))=|t|^{\alpha}+|s|^{\alpha}-|t-s|^{\alpha} .
$$

We note in passing that the first correct proof of Pickands result is given in [27]. It is also well known that $0<H_{\alpha}<\infty$; see [26], [1], [5], [28], [29], [14], [7], [9], [13], [8] , [2], [10] for the main properties of Pickands and related constants. Since

$$
\Psi(u)=\frac{1}{\sqrt{2 \pi} u} \exp \left(-u^{2} / 2\right)(1+o(1)), \text { as } u \rightarrow \infty
$$

it is clear that the results of [4] show that the tail asymptotic behaviour of the supremum of weakly dependent stationary Gaussian processes over random intervals depends on both the tail asymptotic behaviour of $\mathcal{T}$ and that of the supremum of the processes over finite deterministic intervals. Note that our assumptions on $\mathcal{T}$ imply that $\mathrm{e} \mathcal{T}=\infty$. If Assumption (A3) hods for $r=0$, then a strongly dependent stationary Gaussian process boils down to a weakly dependent one. Therefore, it is intuitive to deduce that the results of [4] will still hold for the case $r \in(0, \infty)$. In the main results below, we show that indeed this is the case. The positive constant which additionally appears in the asymptotics is

$$
K_{\lambda, r}=\int_{0}^{\infty} \mathbb{E}(\exp (-x \exp (-r+\sqrt{2 r} \mathcal{W}))) x^{-\lambda} d x \in(0, \infty)
$$

where $\mathcal{W}$ is a $N(0,1)$ random variable.

Theorem 2.1. Let $\{X(t), t \geq 0\}$ be a standard stationary Gaussian processes with correlation function satisfying (A1) and (A3), and let $\mathcal{T}$ be positive random variable independent of $\{X(t), t \geq 0\}$. If $\bar{H}$ satisfies Assumption (B), then we have

$$
P\left(\sup _{t \in[0, \mathcal{T}]} X(t)>u\right)=K_{\lambda, r}\left(\frac{H_{\alpha} C_{*}^{1 / \alpha}}{\sqrt{2 \pi}}\right)^{\lambda} L\left(u^{\frac{\alpha-2}{\alpha}} \exp \left(u^{2} / 2\right)\right) u^{\frac{\lambda(2-\alpha)}{\alpha}} \exp \left(-\frac{\lambda u^{2}}{2}\right)(1+o(1(2) \cdot 4)
$$

as $u \rightarrow \infty$.

Remark 2.1. i) Setting

$$
m(u)=\frac{1}{C_{*}^{1 / \alpha} H_{\alpha} u^{2 / \alpha} \Psi(u)}, \quad u>0
$$

we can re-write Eq. (2.4) as

$$
P\left(\sup _{t \in[0, \mathcal{T}]} X(t)>u\right)=K_{\lambda, r} P(\mathcal{T}>m(u))(1+o(1)), \quad u \rightarrow \infty .
$$

ii) Since $K_{\lambda, 0}=\Gamma(1-\lambda)$, then (2.4) agrees with (1.2) if $r \downarrow 0$. 


\section{FURTHER RESULTS AND PROOF OF THEOREM 2.1}

In this section we present three lemmas and then proceed with the proof of Theorems 2.1.

Let $\left\{X_{i}(t), t \in[0, \infty)\right\}, i=1,2, \cdots$ be independent copies of $\{X(t), t \in[0, \infty)\}$ and $\{\eta(t), t \in$ $[0, \infty)\}$ be such that $\eta(t)=X_{i}(t)$ for $t \in J_{i}=[i-1, i)$. For a fixed constant $T>0$ we define $\rho(T)=r / \log T$ and

$$
\xi_{T}(t)=(1-\rho(T))^{1 / 2} \eta(t)+\rho^{1 / 2}(T) \mathcal{W}, \quad 0 \leq t \leq T,
$$

where $\mathcal{W}$ is a $N(0,1)$ random variable independent of $\{\eta(t), t \in[0, \infty)\}$. Note in passing that $\left\{\xi_{T}(t), t \in\right.$ $[0, T]\}$ is a non-stationary Gaussian process with covariance function $\varrho(s, t)$ given by

$$
\varrho(s, t)=\left\{\begin{array}{cl}
r(s, t)+(1-r(s, t)) \rho(T), & s \in J_{i}, t \in J_{j}, i=j \\
\rho(T), & s \in J_{i}, t \in J_{j}, i \neq j .
\end{array}\right.
$$

Hereafter $a, u$ are positive constants, $k, l$ are integers, and we set

$$
q=q(u)=a u^{-2 / \alpha}, \quad \rho(a)=1-\frac{H_{a}(a)}{H_{a}}, \quad m(u)=\frac{1}{C_{*}^{1 / \alpha} H_{\alpha} u^{2 / \alpha} \Psi(u)}, \quad u>0 .
$$

Further, $\mathcal{C}$ shall denote a positive finite constant whose value may vary from place to place.

In the next two lemmas we shall work with subintervals of $[0, T]$. We divide therefore $[0, T]$ onto intervals of length 1, and split each of them into subintervals $I_{j}^{\varepsilon}, I_{j}$ of length $\varepsilon$ and $1-\varepsilon$, respectively.

Lemma 3.1. Let $a>0$ and $q=q(u)=a u^{-2 / \alpha}$. Suppose that both Assumptions (A1) and (A3) hold. If for some $T=T_{u}$ we have $T / m(u)=O(1)$ as $u \rightarrow \infty$, then

$$
\sum_{\substack{k \in I_{i}, l q \in I_{j} \\ i, j \in\{1,2, \cdots,[T]\}}}|r(k q, l q)-\varrho(k q, l q)| \int_{0}^{1} \frac{1}{\sqrt{1-r^{(h)}(k q, l q)}} \exp \left(-\frac{u^{2}}{1+r^{(h)}(k q, l q)}\right) d h \rightarrow 0
$$

as $T \rightarrow \infty$, where $r^{(h)}(k q, l q)=h r(k q, l q)+(1-h) \varrho(k q, l q)$.

Proof: Let $\vartheta(t)=\sup _{t<|k q-l q| \leq T}\{\varpi(k q, l q)\}$, where $\varpi(k q)=\max \{r(k q, l q), \varrho(k q, l q)\}$. From Assumption (A1), it is easy to see that for any $\varepsilon \in\left(0,2^{-1 / \alpha}\right), \vartheta(\varepsilon)<1$ for all sufficiently large $T$. Consequently, we may choose some positive constant $\beta$ such that $\beta<\frac{1-\vartheta(\varepsilon)}{1+\vartheta(\varepsilon)}$ for all sufficiently large $T$. First, we consider the case that $k q, l q$ are in the same interval $I$, which implies $\varrho(k q, l q)=r(k q, l q)+$ $(1-r(k q, l q)) \rho(T) \sim r(k q,-l q)$ for sufficiently large $T$. Split the left-hand-side of (3.1) into two parts as

$$
\sum_{\substack{k q \in I_{i},, q \in I_{i}, i \in\{1,2, \ldots,[T]\} \\|l q-k q|<\varepsilon}}+\sum_{\substack{k \\ k q \in I_{i}, l q \in I_{i}, i \in\{1,2, \ldots,[T]\} \\ \varepsilon<|l q-k q|<1-\varepsilon}}=: J_{T, 1}+J_{T, 2} .
$$

Note that for $|k q-l q| \in(0, \varepsilon), r(k q, l q)-\varrho(k q, l q)=\rho(T)(1-r(k q, l q))$. By Assumption (A1) for all $|t| \leq \varepsilon<2^{-1 / \alpha}$

$$
\frac{1}{2}|t|^{\alpha} \leq 1-r(t) \leq 2|t|^{\alpha} .
$$

Condition $T / m(u)=O(1)$ implies thus

$$
u^{2}=2 \log T-\log \log T+\frac{2}{\alpha} \log \log T+O(1) .
$$


Consequently, since further $q=a u^{-2 / \alpha}=a(\log T)^{-1 / \alpha}$ we obtain

$$
\begin{aligned}
J_{T, 1} & \leq \mathcal{C} \sum_{\substack{k q \in I_{i}, l q \in I_{i}, i \in\{1,2, \ldots,[T]\} \\
|l q-k q|<\varepsilon}}|r(k q, l q)-\varrho(k q, l q)| \frac{1}{\sqrt{1-r(k q, l q)}} \exp \left(-\frac{u^{2}}{1+r(k q, l q)}\right) \\
& \leq \mathcal{C} \rho(T) \sum_{\substack { k q \in I_{i},{c}{l q \in I_{i}, i \in\{1,2, \ldots,[T]\} \\
|l q-k q|<\varepsilon{ k q \in I _ { i } , \\
\begin{subarray} { c } { l q \in I _ { i } , i \in \{ 1 , 2 , \ldots , [ T ] \} \\
| l q - k q | < \varepsilon } }\end{subarray}} \sqrt{1-r(k q, l q)} \exp \left(-\frac{u^{2}}{1+r(k q, l q)}\right) \\
& \leq \mathcal{C} \rho(T) \frac{T}{q} \sum_{0<k q \leq \varepsilon} \sqrt{1-r(k q)} \exp \left(-\frac{u^{2}}{1+r(k q)}\right) \\
& \leq \mathcal{C} \frac{T}{q} \rho(T) \sum_{0<k q \leq \varepsilon} \sqrt{1-r(k q)} \exp \left(-\frac{u^{2}}{2}\right) \exp \left(-\frac{(1-r(k q)) u^{2}}{2(1+r(k q))}\right) \\
& \leq \mathcal{C} \frac{T}{q} \rho(T) T^{-1}(\log T)^{1 / 2-1 / \alpha} \sum_{0<k q \leq \varepsilon} \sqrt{1-r(k q)} \exp \left(-\frac{(1-r(k q)) u^{2}}{2(1+r(k q))}\right) \\
& \leq \mathcal{C}(\log T)^{-1 / 2} \sum_{0<k q \leq \varepsilon}(k q)^{\alpha / 2} \exp \left(-\frac{1}{8}|k q|^{\alpha}\right) \\
& \leq \mathcal{C}(\log T)^{-1 / 2} .
\end{aligned}
$$

Using the fact that $u \sim(2 \log T)^{1 / 2}$, we have further

$$
\begin{aligned}
& J_{T, 2} \leq \mathcal{C} \sum_{\substack{k \in \in I_{i}, k q \in I_{i}, i \in\{1,2, \ldots,[T]\} \\
\varepsilon<|l q-k q|<1-\varepsilon}}|r(k q, l q)-\varrho(k q, l q)| \exp \left(-\frac{u^{2}}{1+\varpi(k q, l q)}\right) \\
& \leq \mathcal{C} \sum_{\substack{k q \in I_{i}, l q \in \in I_{i}, i \in\{1,2, \ldots,[T]\} \\
\varepsilon<|l q-k q|<1-\varepsilon}} \exp \left(-\frac{u^{2}}{1+\varpi(k q, l q)}\right) \\
& \leq \mathcal{C} \sum_{\substack{k q \in I_{i}, l q \in I_{i}, i \in\{1,2, \ldots,[T]\} \\
\varepsilon<|l q-k q|<1-\varepsilon}} \exp \left(-\frac{u^{2}}{1+\vartheta(\varepsilon)}\right) \\
& \leq \mathcal{C} \frac{T}{q} \exp \left(-\frac{u^{2}}{1+\vartheta(\varepsilon)}\right) \sum_{\varepsilon<k q \leq 1-\varepsilon} 1 \leq \mathcal{C} \frac{T}{q} T^{-\frac{2}{1+\vartheta(\varepsilon)}}(\log T)^{\frac{\alpha-2}{\alpha(1+\vartheta(\varepsilon))}} \sum_{\varepsilon<k q \leq 1-\varepsilon} 1 \\
& \leq \mathcal{C} T^{-\frac{1-\vartheta(\varepsilon)}{1+\vartheta(\varepsilon)}}(\log T)^{\frac{\alpha+2 \vartheta(\varepsilon)}{\alpha(1+\vartheta(\varepsilon))}} .
\end{aligned}
$$

In the second step, we deal with the case that $k q \in I_{i}$ and $l q \in I_{j}, i \neq j$. Note that in this case, the distance between any two intervals $I_{i}$ and $I_{j}$ is larger than $\varepsilon$. We split the left-hand-side of (3.1) into two parts as

$$
\sum_{\substack { k q \in I_{i},{c}{l q \in I_{j}, i \neq j \in\{1,2, \ldots,[T]\} \\
\varepsilon<|l q-k q|<T^{\beta}{ k q \in I _ { i } , \\
\begin{subarray} { c } { l q \in I _ { j } , i \neq j \in \{ 1 , 2 , \ldots , [ T ] \} \\
\varepsilon < | l q - k q | < T ^ { \beta } } }\end{subarray}}+\sum_{\substack{k q \in I_{i}, l q \in I_{j}, i \neq j \in\{1,2, \ldots,[T]\} \\
T^{\beta}<|l q-k q|<T}}=: J_{T, 3}+J_{T, 4} .
$$


Similar to the derivation of (3.5), we have

$$
\begin{aligned}
J_{T, 3} & \leq \mathcal{C} \sum_{\substack { k q \in I_{i}, l \\
\begin{subarray}{c}{l \\
\varepsilon \in I_{j}, i \neq j \in\{1,2, \ldots,[T]\} \\
\varepsilon<|l q-k q|<T^{\beta}{ k q \in I _ { i } , l \\
\begin{subarray} { c } { l \\
\varepsilon \in I _ { j } , i \neq j \in \{ 1 , 2 , \ldots , [ T ] \} \\
\varepsilon < | l q - k q | < T ^ { \beta } } }\end{subarray}}|r(k q, l q)-\varrho(k q, l q)| \exp \left(-\frac{u^{2}}{1+\varpi(k q, l q)}\right) \\
& \leq \mathcal{C} \sum_{\substack { k q \in I_{i}, l \\
\begin{subarray}{c}{q \in \in I_{j}, i \neq j \in\{1,2, \ldots,[T]\} \\
\varepsilon<|l q-k q|<T^{\beta}{ k q \in I _ { i } , l \\
\begin{subarray} { c } { q \in \in I _ { j } , i \neq j \in \{ 1 , 2 , \ldots , [ T ] \} \\
\varepsilon < | l q - k q | < T ^ { \beta } } }\end{subarray}} \exp \left(-\frac{u^{2}}{1+\varpi(k q, l q)}\right) \\
& \leq \mathcal{C} \sum_{\substack{k q \in I_{i}, l q \in I_{j}, i \neq j \in\{1,2, \ldots,[T]\} \\
\varepsilon<|l q-k q|<T^{\beta}}} \exp \left(-\frac{u^{2}}{1+\vartheta(\varepsilon)}\right) \\
& \leq \mathcal{C} \frac{T}{q} \exp \left(-\frac{u^{2}}{1+\vartheta(\varepsilon)}\right) \sum_{\substack{\varepsilon<k q \leq T^{\beta} \\
\exp }} 1 \leq \mathcal{C} \frac{T}{q} T^{-\frac{2}{1+\vartheta(\varepsilon)}}(\log T)^{\frac{\alpha-2}{\alpha(1+\vartheta(\varepsilon))}} \sum_{\varepsilon<k q \leq T^{\beta}} 1 \\
& \leq \mathcal{C} T^{\beta-\frac{1-\vartheta(\varepsilon)}{1+\vartheta(\varepsilon)}(\log T)^{\frac{\alpha+2 \vartheta(\varepsilon)}{\alpha(1+\vartheta(\varepsilon))}} .}
\end{aligned}
$$

Consequently, $\lim _{T \rightarrow \infty} J_{T, 3}=0$ since $\beta<\frac{1-\vartheta(\varepsilon)}{1+\vartheta(\varepsilon)}$.

By Assumption (A3) we have $\vartheta(t) \log t \leq K$ for all large $t$ and some constant $K$. Thus, $\varpi(k q, l q) \leq$ $\vartheta\left(T^{\beta}\right) \leq K / \log T^{\beta}$ for $|k q-l q|>T^{\beta}$. Now using (3.3) again, we obtain

$$
\begin{aligned}
\frac{T^{2}}{q^{2} \log T} \exp \left(-\frac{u^{2}}{1+\vartheta\left(T^{\beta}\right)}\right) & \leq \frac{T^{2}}{q^{2} \log T} \exp \left(-\frac{u^{2}}{1+K / \log T^{\beta}}\right) \\
& \sim \frac{T^{2}}{q^{2} \log T}\left(T^{-2} \log T(\log T)^{-2 / \alpha}\right)^{\frac{1}{1+K / \log T^{\beta}}} \\
& =a^{-2} T^{\left(2 K / \log T^{\beta}\right) /\left(1+K / \log T^{\beta}\right)}(\log T)^{\left((2 / \alpha-1) K / \log T^{\beta}\right) /\left(1+K / \log T^{\beta}\right)} \\
& =O(1) .
\end{aligned}
$$

Next, following the argument of the proof of Lemma 6.4.1 in [20] we have

$$
\begin{aligned}
J_{T, 4} & \leq \mathcal{C} \sum_{\substack{k q \in I_{i}, l_{q \in I_{j}, i \neq j \in\{1,2, \cdots,[T]\}} \\
T^{\beta}<|l q-k q|<T}}|r(k q, l q)-\varrho(k q, l q)| \exp \left(-\frac{u^{2}}{1+\varpi(k q, l q)}\right) \\
& \leq \mathcal{C} \sum_{\substack{k q \in I_{i}, l_{q \in I_{j}, i \neq j \in\{1,2, \cdots,[T]\}} \\
T^{\beta}|l q-k q|<T}}|r(k q, l q)-\rho(T)| \exp \left(-\frac{u^{2}}{1+\vartheta\left(T^{\beta}\right)}\right) \\
& \leq \mathcal{C} \frac{T}{q} \exp \left(-\frac{u^{2}}{1+\vartheta\left(T^{\beta}\right)}\right) \sum_{T^{\beta}<k q \leq T}|r(k q)-\rho(T)| \\
& \leq \mathcal{C} \frac{T^{2}}{q^{2} \log T} \exp \left(-\frac{u^{2}}{1+K / \log T^{\beta}}\right) \times \frac{q \log T}{T} \sum_{T^{\beta}<k q \leq T}|r(k q)-\rho(T)| \\
& \leq \mathcal{C} \frac{q \log T}{T} \sum_{T^{\beta}<k q \leq T}|r(k q)-\rho(T)| \\
& \leq \mathcal{C} \frac{q}{\beta T} \sum_{T^{\beta}<k q \leq T}|r(k q) \log k q-r|+\mathcal{C} r \frac{q}{T} \sum_{T^{\beta}<k q \leq T}\left|1-\frac{\log T}{\log k q}\right| .
\end{aligned}
$$


Using again Assumption (A3) it follows that the first term of (3.8) tends to 0 as $T \rightarrow \infty$. Furthermore, the second term of (3.8) also tends to 0 by an estimate as in the proof of Lemma 6.4.1 in [20]. The proof follows now from (3.2)-(3.8).

The next lemma is consequence of Lemma 12.2.11 in [20].

Lemma 3.2. Let $h>0$ be a fixed constant such that $\sup _{\varepsilon \leq t \leq h} r(t)<1$ for each $\varepsilon>0$, and set $q(u)=a u^{2 / \alpha}$. If Assumption (A1) holds, then for each interval I of length $h$ we have

$$
0 \leq P\left(\max _{k q \in I} X(k q) \leq u\right)-P\left(\sup _{s \in I} X(s) \leq u\right) \leq h \rho(a) \frac{1}{m(u)}+o\left(\frac{1}{m(u)}\right)
$$

where $\rho(a) \rightarrow 0$ as $a \downarrow 0$.

Lemma 3.3. Let $\{X(t), t \geq 0\}$ be a centered stationary Gaussian processes with covariance function $r(t)$ satisfying (A1) and (A3). Then for any $0<A_{0}<A_{\infty}<\infty$

$$
P\left(\sup _{s \in[0, x m(u)]} X(s) \leq u\right) \rightarrow \mathbb{E}(\exp (-x \exp (-r+\sqrt{2 r} \mathcal{W}))) \in(0, \infty)
$$

is valid as $u \rightarrow \infty$ uniformly for $x \in\left[A_{0}, A_{\infty}\right]$, with $\mathcal{W}$ a standard Gaussian random variable.

Proof: Let $n_{x}:=[x m(u)]$, and fix some $\varepsilon>0$. Divide the interval $\left[0, n_{x}\right]$ onto intervals of length 1 , and split each of them into subintervals $I_{j}^{\varepsilon}, I_{j}$ of length $\varepsilon$ and $1-\varepsilon$, respectively. In the first step, we show that

$$
P\left(\sup _{s \in[0, x m(u)]} X(s) \leq u\right)-P\left(\sup _{s \in \cup I_{j}} X(s) \leq u\right) \rightarrow 0
$$

and

$$
P\left(\sup _{s \in[0,1]} X(s) \leq u\right)-P\left(\sup _{s \in I_{1}} X(s) \leq u\right) \rightarrow 0
$$

as $u \rightarrow \infty$ and $\varepsilon \downarrow 0$, where for (3.9) the convergence holds uniformly for $x \in\left[A_{0}, A_{\infty}\right]$. Making use of the stationarity of $\{X(t), t \geq 0\}$ and the Pickands exact asymptotics, we obtain

$$
\begin{aligned}
\left|P\left(\sup _{s \in[0, x m(u)]} X(s) \leq u\right)-P\left(\sup _{s \in \cup I_{j}} X(s) \leq u\right)\right| & \leq \sum_{j=1}^{n_{x}} P\left(\sup _{s \in I_{j}^{\varepsilon}} X(s)>u\right) \\
& =n_{x} \varepsilon C_{*}^{1 / \alpha} H_{\alpha} u^{1 / \alpha} \Psi(u)(1+o(1)) \\
& =x \varepsilon(1+o(1)) \\
& \leq A_{\infty} \varepsilon(1+o(1)) \rightarrow 0
\end{aligned}
$$

as $u \rightarrow \infty$ and $\varepsilon \downarrow 0$, hence (3.9) follows. Since the proof of (3.10) follows with similar arguments, we omit it.

In the second step, we prove that

$$
P\left(\sup _{s \in \cup I_{j}} X(s) \leq u\right)-P\left(\max _{k q \in \cup I_{j}} X(k q) \leq u\right) \rightarrow 0
$$


and

$$
P\left(\sup _{s \in I_{1}} X(s) \leq u\right)-P\left(\max _{k q \in I_{1}} X(k q) \leq u\right) \rightarrow 0
$$

as $u \rightarrow \infty$ and $a \downarrow 0$, uniformly for $x \in\left[A_{0}, A_{\infty}\right]$. Next, by Lemma 3.2

$$
\begin{aligned}
\left|P\left(\sup _{s \in \cup I_{j}} X(s) \leq u\right)-P\left(\max _{k q \in \cup I_{j}} X(k q) \leq u\right)\right| \leq & n_{x} \max _{j}\left(P\left(\max _{k q \in I_{j}} X(k q) \leq u\right)-P\left(\sup _{s \in I_{j}} X(s) \leq u\right)\right) \\
& \leq(1-\varepsilon) \frac{n_{x}}{m(u)} \rho(a)+n_{x} o\left(\frac{1}{m(u)}\right) \\
& \leq(1-\varepsilon) A_{\infty} \rho(a)+A_{\infty} m(u) o\left(\frac{1}{m(u)}\right) \rightarrow 0
\end{aligned}
$$

as $u \rightarrow \infty$ and $a \downarrow 0$, which completes the proof of (3.11). We omit the proof of (3.12) since it is similar to that of (3.11).

In the third step we show that

$$
P\left(\max _{k q \in \cup I_{j}} X(k q) \leq u\right)-P\left(\max _{k q \in \cup I_{j}} \xi_{n_{x}}(k q) \leq u\right) \rightarrow 0
$$

as $u \rightarrow \infty$, uniformly for $x \in\left[A_{0}, A_{\infty}\right]$. Applying Theorem 4.2.1 in [20], along similar lines to the proof of Theorem 8.2.4 therein we obtain

$$
\begin{aligned}
& \left|P\left(\max _{k q \in \cup I_{j}} X(k q) \leq u\right)-P\left(\max _{k q \in \cup I_{j}} \xi_{n_{x}}(k q) \leq u\right)\right| \\
& \leq \sum_{\substack{k q \in I_{i}, l q \in I_{j} \\
i, j \in\left\{1,2, \ldots, n_{x}\right\}}}|r(k q, l q)-\varrho(k q, l q)| \int_{0}^{1} \frac{1}{\sqrt{1-r^{(h)}(k q, l q)}} \exp \left(-\frac{u^{2}}{1+r^{(h)}(k q, l q)}\right) d h,
\end{aligned}
$$

where $r^{(h)}(k q, l q)=h r(k q, l q)+(1-h) \varrho(k q, l q)$. Note that $n_{x} / m(u) \sim x \in\left(A_{0}, A_{\infty}\right)$, hence (3.13) follows applying Lemma 3.1. Next, in view of the definition of $\left\{\xi_{n_{x}}(t), 0 \leq t \leq n_{x}\right\}$, we have

$$
\begin{aligned}
P\left(\max _{k q \in \cup I_{j}} \xi_{n_{x}}(k q) \leq u\right) & =P\left(\max _{k q \in \cup I_{j}}\left(\left(1-\rho\left(n_{x}\right)\right)^{1 / 2} \eta(k q)+\rho^{1 / 2}\left(n_{x}\right) \mathcal{W}\right) \leq u\right) \\
& =P\left(\left(1-\rho\left(n_{x}\right)\right)^{1 / 2}\left(\max _{k q \in \cup I_{j}} \eta(k q)\right)+\rho^{1 / 2}\left(n_{x}\right) \mathcal{W} \leq u\right) \\
& =\int_{-\infty}^{\infty} P\left(\max _{k q \in \cup I_{j}} \eta(k q) \leq \frac{u-\rho^{1 / 2}\left(n_{x}\right) z}{\left(1-\rho\left(n_{x}\right)\right)^{1 / 2}}\right) d \Phi(z),
\end{aligned}
$$

with $\Phi$ the standard Gaussian distribution function on $\mathbb{R}$. As $u \rightarrow \infty$ we have

$$
u_{z}:=\frac{u-\rho^{1 / 2}\left(n_{x}\right) z}{\left(1-\rho\left(n_{x}\right)\right)^{1 / 2}}=u+\frac{-\sqrt{2 r} z+r}{u}+o\left(\frac{1}{u}\right), \quad \frac{1}{m\left(u_{z}\right)}=\frac{e^{-r+\sqrt{2 r} z}}{m(u)}(1+o(1)) .
$$


Hence, the definition of $\{\eta(t), t \geq 0\}$ and (3.10),(3.12) imply

$$
\begin{aligned}
P\left(\max _{k q \in \cup I_{j}} \eta(k q) \leq u_{z}\right) & =\prod_{j=1}^{n_{x}} P\left(\max _{k q \in I_{j}} X_{j}(k q) \leq u_{z}\right)(1+o(1)) \\
& =\left(P\left(\max _{k q \in I_{1}} X(k q) \leq u_{z}\right)\right)^{n_{x}}(1+o(1)) \\
& =\left(P\left(\sup _{t \in I_{1}} X(t) \leq u_{z}\right)\right)^{n_{x}}(1+o(1)) \\
& =\left(P\left(\sup _{t \in[0,1]} X(t) \leq u_{z}\right)\right)^{n_{x}}(1+o(1)) \\
& =\left(1-\frac{1}{m\left(u_{z}\right)}+o\left(\frac{1}{m\left(u_{z}\right)}\right)\right)^{x m(u)}(1+o(1)) \\
& =\left(1-\frac{e^{-r+\sqrt{2 r} z}}{m(u)}+o\left(\frac{1}{m(u)}\right)\right)^{x m(u)}(1+o(1)) \\
& =\exp \left(-e^{-r+\sqrt{2 r} z} x\right)(1+o(1))
\end{aligned}
$$

uniformly for $x \in\left[A_{0}, A_{\infty}\right]$, as $u \rightarrow \infty$. Combining the last result with (3.9),(3.11),(3.13),(3.14) and applying the dominated convergence theorem completes the proof.

Proof of THEOREM 2.1. Consider first the case $\lambda>0$. For any two positive constants $A_{0}<A_{\infty}$ we may write

$$
\begin{aligned}
P\left(\sup _{s \in[0, \mathcal{T}]} X(s)>u\right) & =\int_{0}^{A_{0} m(u)} P\left(\sup _{s \in[0, t]} X(s)>u\right) d H(t)+\int_{A_{0} m(u)}^{A_{\infty} m(u)} P\left(\sup _{s \in[0, t]} X(s)>u\right) d H(t) \\
& +\int_{A_{\infty} m(u)}^{\infty} P\left(\sup _{s \in[0, t]} X(s)>u\right) d H(t)=: S_{1}+S_{2}+S_{3},
\end{aligned}
$$

with $H$ the distribution function of $\mathcal{T}$. From the proof of Theorem 3.2 in [4] for all $u$ large we have

$$
S_{1} \leq \frac{\lambda}{1-\lambda} A_{0}^{1-\lambda} P(\mathcal{T}>m(u))(1+o(1))
$$

and

$$
S_{3} \leq P\left(T>A_{\infty} m(u)\right)=A_{\infty}^{-\lambda} P(\mathcal{T}>m(u))(1+o(1))
$$

For $\varepsilon>0$ and $u$ sufficiently large Lemma 3.3 implies the following (write $\phi$ for the density function of $\Phi)$ upper bound

$$
\begin{aligned}
\frac{S_{2}}{1+\epsilon} & =\frac{1}{1+\epsilon} \int_{A_{0}}^{A_{\infty}} P\left(\sup _{s \in[0, x m(u)]} X(s)>u\right) d H(x m(u)) \\
& \leq \int_{A_{0}}^{A_{\infty}}\left(1-\int_{-\infty}^{\infty} \exp \left(-e^{-r+\sqrt{2 r} z} x\right) \phi(z) d z\right) d H(x m(u)) \\
& =C_{A_{0}, A_{\infty}}-\left(1-A_{\infty}^{(r)}\right) P\left(\mathcal{T}>A_{\infty} m(u)\right)+\left(1-A_{0}^{(r)}\right) P\left(\mathcal{T}>A_{0} m(u)\right),
\end{aligned}
$$


where

$$
C_{A_{0}, A_{\infty}}=\int_{A_{0}}^{A_{\infty}} \int_{-\infty}^{\infty} \exp \left(-e^{-r+\sqrt{2 r} z} x\right) \phi(z) P(\mathcal{T}>x m(u)) d x d z, \quad A^{(r)}=\operatorname{eexp}\left(-e^{-r+\sqrt{2 r} \mathcal{W}} A\right) .
$$

In an analogous way for all $u$ large

$$
\frac{S_{2}}{1-\epsilon} \geq C_{A_{0}, A_{\infty}}-\left(1-A_{\infty}^{(r)}\right) P\left(\mathcal{T}>A_{\infty} m(u)\right)+\left(1-A_{0}^{(r)}\right) P\left(\mathcal{T}>A_{0} m(u)\right) .
$$

In view of Assumption (B) and Theorem 1.5.2 in [6]

$$
C_{A_{0}, A_{\infty}}=P(\mathcal{T}>m(u)) \int_{A_{0}}^{A_{\infty}} \int_{-\infty}^{\infty} \exp \left(-e^{-r+\sqrt{2 r} z} x\right) x^{-\lambda} \phi(z) d x d z(1+o(1)), \quad u \rightarrow \infty .
$$

Consequently, letting $A_{0} \rightarrow 0, A_{\infty} \rightarrow \infty$ and $\epsilon \rightarrow 0$, we conclude that both $S_{1}$ and $S_{3}$ are negligible compared to $S_{2}$, and furthermore

$$
S_{2}=\int_{0}^{\infty} \int_{-\infty}^{\infty} \exp \left(-e^{-r+\sqrt{2 r} z} x\right) x^{-\lambda} \phi(z) d x d z P(\mathcal{T}>m(u))(1+o(1))
$$

as $u \rightarrow \infty$. Consequently Assumption (B), the definition of $m(u)$ and (2.2) complete the proof.

Next we consider the case $\lambda=0$. As in the proof of Theorem 3.3 in [4] for given $A_{\infty}>0$ Lemma 3.3 implies

$$
\begin{aligned}
P\left(\sup _{s \in[0, \mathcal{T}]} X(s)>u\right) & \geq \int_{A_{\infty} m(u)}^{\infty} P\left(\sup _{s \in[0, t]} X(s)>u\right) d H(t) \\
& \geq P\left(\sup _{s \in\left[0, A_{\infty} m(u)\right]} X(s)>u\right) P\left(\mathcal{T}>A_{\infty} m(u)\right)(1+o(1)) \\
& =\left(1-A_{\infty}^{(r)}\right) P(\mathcal{T}>m(u))(1+o(1))
\end{aligned}
$$

as $u \rightarrow \infty$. Thus, letting $A_{\infty} \rightarrow \infty$, we get that

$$
P\left(\sup _{s \in[0, \mathcal{T}]} X(s)>u\right) \geq P(\mathcal{T}>m(u))(1+o(1))
$$

as $u \rightarrow \infty$. Furthermore,

$$
\begin{aligned}
P\left(\sup _{s \in[0, \mathcal{T}]} X(s)>u\right) & \leq \int_{0}^{A_{0} m(u)} P\left(\sup _{s \in[0, t]} X(s)>u\right) d H(t)+P\left(\mathcal{T}>A_{0} m(u)\right) \\
& \leq P\left(\sup _{s \in[0,1]} X(s)>u\right)\left[\int_{0}^{A_{0} m(u)} P(\mathcal{T}>t) d t+1\right]+P\left(\mathcal{T}>A_{0} m(u(\mathcal{B}) .16)\right.
\end{aligned}
$$

Applying Karamata's theorem (see, e.g., Proposition 1.5.8 in [6]) we have, as $u \rightarrow \infty$

$$
\int_{0}^{A_{0} m(u)} P(\mathcal{T}>t) d t=A_{0} m(u) P\left(\mathcal{T}>A_{0} m(u)\right)(1+o(1))
$$


which combined with (3.16) and Theorem D.2 in [28] yields

$$
P\left(\sup _{s \in[0, \mathcal{T}]} X(s)>u\right) \leq\left(1+A_{0}\right) P(\mathcal{T}>m(u))(1+o(1))
$$

as $u \rightarrow \infty$. The claim follows now letting $A_{0} \rightarrow 0$.

Acknowledgement: The authors would like to thank the referee, the Editor-in-Chief and Professor K. Dȩbicki for several suggestions which improved this paper. Both authors kindly acknowledge support by the Swiss National Science Foundation Grant 200021-1401633/1.

\section{REFERENCES}

1. RJ Adler, An introduction to continuity, extrema and related topics for general gaussian processes.—ims lect, Notes, Monograph Series, 12, 1990.

2. JMP Albin and H. Choi, A new proof of an old result by pickands, Electronic Communications in Probability, 15:339-345, 2010.

3. M. Arendarczyk and K. Dębicki, Asymptotics of supremum distribution of a gaussian process over a weibullian time, Bernoulli, 17(1):194-210, 2011.

4. M. Arendarczyk and K. Dębicki, Exact asymptotics of supremum of a stationary gaussian process over a random interval, Statistics \& Probability Letters, 2011.

5. M.S. Berman, Sojourns and extremes of stochastic processes. 1992, Wadsworth \& Brooks/Cole, Boston.

6. N.H. Bingham, C.M. Goldie, and J.L. Teugels, Regular variation, Volume 27, Cambridge university press, 1989.

7. K. Dẹbicki, Ruin probability for gaussian integrated processes, Stochastic Processes and their Applications, 98(1):151-174, 2002.

8. K. Dębicki and P. Kisowski, A note on upper estimates for pickands constants, Statistics \& Probability Letters, 78(14):2046-2051, 2008.

9. K. Dębicki, Z. Michna, and T. Rolski, Simulation of the asymptotic constant in some fluid models, Stochastic Models, 19(3):407-423, 2003.

10. K. Dębicki and K. Tabiś, Extremes of the time-average of stationary gaussian processes, Stochastic Processes and their Applications, 121(9):2049-2063, 2011.

11. K. Dębicki and M. Uitert, Large buffer asymptotics for generalized processor sharing queues with gaussian inputs, Queueing Systems, 54(2):111-120, 2006.

12. K. Dębicki, B. Zwart, and S. Borst, The supremum of a gaussian process over a random interval, Statistics \& probability letters, 68(3):221-234, 2004.

13. AB Dieker, Extremes of gaussian processes over an infinite horizon, Stochastic Processes and Their Applications, 115(2):207-248, 2005.

14. E. Hashorva and J. Hüsler, Extremes of gaussian processes with maximal variance near the boundary points, Methodology and Computing in Applied Probability, 2(3):255-269, 2000.

15. E. Hashorva and Z. Weng, Limit laws for extremes of dependent stationary gaussian arrays, Statistics \& Probability Letters, pp. -, 2013.

16. H.C. Ho and W.P. McCormick, Asymptotic distribution of sum and maximum for gaussian processes, Journal of Applied Probability, 36(4):1031-1044, 1999.

17. Z. Kabluchko, Extremes of independent gaussian processes, Extremes, 14(3):285-310, 2011. 
18. DG Konstantinides, V. Piterbarg, and S. Stamatovic, Gnedenko-type limit theorems for cyclostationary $\chi^{2}$-processes, Lithuanian Mathematical Journal, 44(2):157-167, 2004.

19. A.V. Kudrov and V.I. Piterbarg, On maxima of partial samples in gaussian sequences with pseudo-stationary trends, Lithuanian Mathematical Journal, 47(1):48-56, 2007.

20. MR Leadbetter, G. Lindgren, and H. Rootzén, Extremes and related properties of random sequences and processes, Volume 11, Springer Verlag, 1983.

21. W.V. Li and Q.M. Shao, Gaussian processes: inequalities, small ball probabilities and applications, Stochastic Processes: Theory and Methods, 19:533-597, 2001.

22. MA Lifshits, Gaussian random functions, Kluwer Academic Publishers (Dordrecht and Boston), 1995.

23. Y. Liu and Q. Tang, The subexponential product convolution of two weibull-type distributions, Journal of the Australian Mathematical Society, 89:277-288, 2010.

24. Y. Mittal and D. Ylvisaker, Limit distributions for the maxima of stationary gaussian processes, Stochastic Processes and their Applications, 3(1):1-18, 1975.

25. Z. Palmowski and B. Zwart, Tail asymptotics of the supremum of a regenerative process, Journal of Applied Probability, 44(2):349-365, 2007.

26. J. Pickands, Asymptotic properties of the maximum in a stationary gaussian process, Trans. Amer. Math. Soc, 145:75-86, 1969.

27. V.I. Piterbarg, On the paper by J. Pickands "upcrosssing probabilities for stationary gaussian processes", Vestnik Moscow Univ. Ser. I Mat. Mekh. 27, 25-30. English transl. in Moscow Univ. Math. Bull. 1972,27, 27:25-30, 1972.

28. V.I. Piterbarg, Asymptotic methods in the theory of Gaussian processes and fields, Volume 148, American Mathematical Soc., 1996.

29. Q.M. Shao, Bounds and estimators of a basic constant in extreme value theory of gaussian processes, Statistica Sinica, 6:245-258, 1996.

30. B. Stamatovic and S. Stamatovic, Cox limit theorem for large excursions of a norm of a gaussian vector process, Statistics \& probability letters, 80(19):1479-1485, 2010.

31. Z. Tan and E. Hashorva, On piterbarg max-discretisation theorem for standardised maximum of stationary gaussian processes, Methodology and Computing in Applied Probability, in press, DOI 10.1007/s11009-012-9305-8, 2012.

32. Z. Tan and E. Hashorva, Limit theorems for extremes of strongly dependent cyclo-stationary $\chi$-processes, Extremes, in press, DOI 10.1007/s10687-013-0170-9, 2013.

33. Z. Tan, E. Hashorva, and Z. Peng, Asymptotics of maxima of strongly dependent gaussian processes, Journal of applied probability, 49:1106-1118, 2012.

34. Z. Tan and Y. Wang, Extremes values of discrete and continuous time strongly dependent gaussian processes, Communications in Statistics - Theory and Methods, in press, DOI 10.1080/03610926.2011.611322, 2012.

35. B. Zwart, S. Borst, and K. Dębicki, Subexponential asymptotics of hybrid fluid and ruin models, The Annals of Applied Probability, 15(1A):500-517, 2005. 